\title{
State of the art of aldosterone immunoassays. A multicenter collaborative study on the behalf of the Cardiovascular Biomarkers Study Group of the Italian Section of European Society of Ligand Assay (ELAS) and Società Italiana di Biochimica Clinica (SIBIOC)
}

\author{
Antonio Fortunato $^{\mathrm{a}, *}$, Concetta Prontera ${ }^{\mathrm{b}}$, Silvia Masotti ${ }^{\mathrm{b}}$, Maria Franzini ${ }^{\mathrm{b}}$, Cristina Marchetti ${ }^{\mathrm{a}}$, \\ Stefania Giovannini ${ }^{\mathrm{c}}$, Gian Carlo Zucchelli ${ }^{\mathrm{c}}$, Michele Emdin ${ }^{\mathrm{b}}$, Claudio Passino ${ }^{\mathrm{b}}$, Aldo Clerico ${ }^{\mathrm{b}}$ \\ a Clinical Chemistry and Hematology Laboratory, San Bortolo Hospital, Viale F. Rodolfi, 37, Vicenza, Italy \\ ${ }^{\mathrm{b}}$ Department of Cardiovascular Medicine and Laboratory Medicine, Fondazione CNR-Regione Toscana G. Monasterio, Via G. Moruzzi 1, Pisa, Italy \\ c QualiMedLab srl, Via G. Moruzzi 1, Pisa, Italy
}

\section{A R T I C L E I N F O}

\section{Article history:}

Received 25 May 2014

Received in revised form 14 January 2015

Accepted 23 January 2015

Available online 4 February 2015

\section{Keywords:}

Aldosterone

Immunoassays methods

RIA

External quality control

\begin{abstract}
A B S T R A C T
Background: Two new immunoassay methods for aldosterone assay using automated platforms recently became available into market. The main aim of the present study is to evaluate the analytical performance of these automated direct immunoassay methods, and also to compare their analytical characteristics to those of the most popular RIA and EIA methods used in an Italian External Quality Assessment (EQA) study.

Methods: In this study analytical performances of two aldosterone immunoassays using the IDS iSYS and DiaSorin LIAISON fully automated platforms, were evaluated. Results obtained with the two platforms in EDTA plasma samples of healthy subjects and patients were compared with those obtained by RIA and EIA methods used in the Italian EQA scheme, named Immunocheck study.

Results: The two automated methods showed similar analytical performances: LoD 83.9 vs 92.2 pmol/L, LoQ 104.4 vs $111.1 \mathrm{pmol} / \mathrm{L}$, respectively; moreover, the within-run and total imprecision values showed CV\% between 8.1 and 14.1 for samples with 180.8 and $387.2 \mathrm{pmol} / \mathrm{L}$ concentration for both methods. There was a close linear regression between methods, however we found a significant proportional bias between LIAISON and iSYS methods. The EQA samples results obtained with these two methods were highly correlated to the consensus mean values.

Conclusions: Our data indicate that aldosterone values measured with the two automated methods actually show better reproducibility, shorter laboratory Turn Around Time (TAT) and require less "hands on labor" compared to RIA and EIA immunoassays. However, in our study significant bias was observed in result comparison, this means that translating aldosterone concentration in clinical information an appropriate definition of reference ranges for each method is mandatory.
\end{abstract}

(C) 2015 Elsevier B.V. All rights reserved.

\section{Introduction}

Aldosterone is a corticosteroid hormone produced by the outer section (zona glomerulosa) of the adrenal cortex. Recent studies reported that also other tissues than adrenal gland (such as vasculature and heart) may produce aldosterone [1]. The limited studies that have been performed suggest that aldosterone's regulation in these tissues,

\footnotetext{
* Corresponding author at: Laboratorio di Chimica Clinica ed Ematologia, Ospedale San Bortolo, Viale F. Rodolfi, 37, 36100 Vicenza, Italy. Tel./fax: +39 0444753230.

E-mail addresses: antonio.fortunato@ulssvicenza.it (A. Fortunato), tina@ftgm.it (C. Prontera), silvi87@gmail.com (S. Masotti), franzinimaria@gmail.com (M. Franzini), cristina.marchetti@ulssvicenza.it (C. Marchetti), stefania@ifc.cnr.it (S. Giovannini), zucchell@ifc.cnr.it (G.C. Zucchelli), emdin@ftgm.it (M. Emdin), passino@ftgm.it (C. Passino), aldo.clerico@ftgm.it (A. Clerico).
}

may be under the same control as aldosterone production by the adrenal cortex [1].

From a clinical point of view, the aldosterone assay is recommended by international guidelines mainly for screening and diagnosis of primary aldosteronism [2-4]. Recent data suggest that primary aldosteronism is present in about $10 \%$ of patients with primary arterial hypertension, in particular those with the so-called low-renin hypertension syndrome, characterized by arterial hypertension, hypokalemia and circulating low renin levels.

From an analytical point of view, aldosterone measurement in blood or urine samples actually has some analytical critical points due to the low amount of the steroid and the presence of a lot of possible interfering substances in body fluids. Competitive immunoassays are the methods usually used for the measurement of aldosterone. About 
40 years ago, the first immunoassays set up for the aldosterone assay were RIA methods [5], which usually included a preliminary extraction and/or chromatographic purification step in order to improve the analytical sensitivity and accuracy [6]. More recently, direct (without extraction or purification step) immunoassay competitive methods, using antisera and monoclonal antibodies with higher affinity and specificity for the steroid molecule and non-isotopic labels, were set up for the measurement of aldosterone in all body fluids, including also saliva [6-10].

Accuracy of aldosterone measurement in biological fluids can be improved using several gas-chromatographic (GS) or high-performance liquid chromatographic (HPLC) mass spectrometry (MS) techniques [11-13]. Although these techniques are considered a reference method that provides both accurate results and excellent specificity, these methods are more expensive and time consuming, and less practicable than immunoassay methods. Furthermore, these methods usually require sample preparation, such as chemical derivatization, and dedicated laboratory staff. Therefore, due to the lack of automation and complexity, GS-MS and HPLC-MS methods are not actually used by the majority of the routine clinical services [12]. In fact, the data from inter-laboratory quality control surveys (External Quality Control: EQA) show that only very few laboratories use MS: e.g. in the survey set RAP (specific control for Aldosterone and Renin) of the College of American Pathologists (CAP) program only a RIA method is used for at least 20 laboratories (the minimum number of results for a statistical significance) and, among 86 laboratories participating to an Italian national program [i.e., Immunocheck program by QualiMedLab (http://www. qualimedlab.it)], none reports Aldosterone measurement performed by MS.

Very recently, some immunoassay methods for aldosterone assay, using fully automated platforms, became available into market; these new methods should share analytical performances better than the previous competitive RIA and EIA methods. The main aim of the present study is to evaluate the analytical performances of two automated direct immunoassay systems, which recently became commercially available (i.e., LIAISON platform by DiaSorin, Saluggia, Italy, and iSYS platform by IDS Ltd., Boldon, UK) in terms of reproducibility of measurements and correlation of results obtained with both the automated analyzers with RIA and EIA methods. The second aim is to evaluate the differences of results obtained in the two referral laboratories, using these automated immunoassay systems in the same clinical samples, collected from healthy subjects and patients with cardiovascular disease and/or hyperaldosteronism. Furthermore, to assess the accuracy (true value) of measurements, the data produced with the most used immunoassay methods by all participant laboratories to an Italian EQA scheme were compared with those measured with the two automated systems in some control samples.

\subsection{Materials and methods}

\subsubsection{Assay methods}

The reproducibility of measurements, within and between assays, and feasibility of two direct immunoassays using automated platforms were independently evaluated in two referral Italian laboratories (i.e., the Department of Laboratory Medicine, Fondazione CNR-Regione Toscana G. Monasterio, Pisa, and Clinical Chemistry Laboratory, San Bortolo Hospital, Vicenza).

One of the automated immunoassay system evaluated for aldosterone assay was the competitive chemiluminescence assay using LIAISON platform (code 310450, DiaSorin, Saluggia, Italy). The principle components of this immunoassay consist of magnetic particles coated with anti-sheep antibody that bind the sheep anti-aldosterone monoclonal antibody. An aldosterone labeled conjugate containing an isoluminol derivative competes with steroid hormone from the calibrators, control and patient samples. The light signal is measured as relative light unit (RLU), which is inversely proportional to the concentration of aldosterone present in calibrators, control or patient samples. The laboratory Turn Around Time (TAT), understood as the time to complete the analytical procedure, is less than $60 \mathrm{~min}$. According to the manufacturer's instructions the measurable range is $83.1-2770 \mathrm{pmol} / \mathrm{L}$ and any value that reads below $83.1 \mathrm{pmol} / \mathrm{L}$ [14].

The analytical performance of the IDS-iSYS aldosterone method (code IS-3300) using the iSYS platform (IDS Ltd., Boldon, UK) was also evaluated in the two study referral laboratories. This immunoassay system is based on chemiluminescent technology. A biotinylated monoclonal anti-aldosterone antibody is incubated with the sample, after an incubation step and aldosterone acridinium conjugate is added and, after a further incubation step, streptavidin coated magnetic particles are added. Following a third incubation step, the antibody-aldosterone complex was separated by the unbound aldosterone using a magnet. After a washing step and addition of trigger reagents, the light emitted by the acridinium label is inversely proportional to the concentration of aldosterone in the original sample. The TAT is less than $60 \mathrm{~min}$. According to the manufacturer's instructions for bulletin use (REF IS-3300) the reportable range of the IDS-iSYS aldosterone method is 102.5-3656 pmol/1 [15].

The performances of these two automated immunoassay systems were compared to those of some RIA methods for aldosterone in the two referral laboratories. The first RIA method evaluated was the kit RIA ALDOCTK-2 (code P2714/100 tubes, DiaSorin, Saluggia, Italy). The second RIA method used was Aldosterone Coat-A-Count (Siemens, Los Angeles USA). The principle of both RIA methods is based on the competition between ${ }^{125}$ I-labeled aldosterone and steroid molecules contained in calibrators or samples to be assayed. These competitive immunoassays are based on the use of antibody-coated tubes for $\mathrm{B} / \mathrm{F}$ separation. The TAT is about $22-24 \mathrm{~h}$ (i.e., using overnight incubation) for both RIA assays.

\subsubsection{Analytical performance of automated immunoassay methods}

The analytical performances of the two automated immunoassays were determined in accordance with the CLSI EP17-A protocol [15] for evaluating the limits of blank (LoB) and detection (LoD), the CLSI EP5A2 protocol [16] for the assay reproducibility by repeatedly measuring 2 different EDTA plasma samples throughout 20 consecutive working days. These evaluations were carried out separately in the two referral laboratories using different sets of samples. The LoB value was assessed by measuring the saline solution (considered as the blank of the method) in 5 different runs for 65 times using two different lots of calibrators. The RLU value corresponding to LoB was calculated according to the formula $\mathrm{RLU}_{\mathrm{LoB}}=$ mean value $-1.645 \mathrm{SD}$ [15]. The LoD was calculated according to the formula: $\mathrm{LoD}=\mathrm{LoB}+1.645 \mathrm{SD}$ [16], where SD is the standard deviation of measured values of a sample pool with very low aldosterone concentration. The between-runs imprecision profile was performed by repeatedly measuring several EDTA blood samples collected from healthy subjects and patients in 20 different runs. In particular, in the Laboratory of Fondazione G. Monasterio, the aldosterone assay analytical performances using LIAISON platform were evaluated and those of automated iSYS platform (IDS - Boldon, UK) were evaluated at "San Bortolo" hospital laboratory in Vicenza.

\subsubsection{Clinical samples}

The referral laboratories collected samples from healthy subjects or patients for the evaluation of analytical performances and the comparison of clinical results obtained with the two automated immunoassay systems. All subjects enrolled have released an informed consent.

In the clinical ward of the Fondazione G. Monasterio, 367 blood samples (8-10 mL) of 168 apparently healthy subjects and 199 patients with cardiovascular disease were collected in EDTA polypropylene tubes between 08:00 and 09:00 a.m. after overnight fast in supine position at rest for at least $15 \mathrm{~min}$. After centrifugation at $3000 \times \mathrm{g}$ for $10 \mathrm{~min}$, the plasma was collected in various aliquots of $0.5 \mathrm{~mL}$, put in polypropylene, and finally stored at $-20{ }^{\circ} \mathrm{C}$ until the assay. Healthy 
subjects were enrolled in the study after an accurate clinical examination, laboratory tests, ECG and cardiac imaging analysis (echocardiography). We excluded all subjects presenting cardiac or systemic acute or chronic diseases, such as previous myocardial infarction, heart failure, coronary heart disease, hypertension, diabetes, kidney disease, obesity, tumor, hepatitis, and pulmonary diseases. Subjects using drugs up to two weeks before aldosterone assay, except for substitutive hormonal therapy, were also excluded.

At San Bortolo Hospital plasma EDTA samples were collected, from 20 apparently healthy male and female volunteers, aged 21-65 years, with normal blood pressure and fasting glucose levels. The exclusion criteria were a need for prescription medications or a physicianprescribed restricted diet, pregnancy, breastfeeding, and the use of oral contraceptives. The blood samples were drawn between 7.00 a.m. and 12.00 a.m. from subjects in a standing or supine position: the standing samples were drawn after the subjects had been walking for $2 \mathrm{~h}$, and the supine samples were drawn after the subjects had been lying supine for at least $1 \mathrm{~h}$. The samples were handled in the same way previously described. Moreover, the Aldosterone Coat-A-Count and ALDOCTK-2 RIA methods were used to compare results obtained with iSYS system in 220 remnant plasma EDTA samples from routine activity; the samples were stored at $-80{ }^{\circ} \mathrm{C}$ and then reassayed in the same day with all the methods in evaluation. Urinary aldosterone measurements, performed with iSYS system and ALDOCTK-2 RIA, were evaluated in 88 remnant samples of $24 \mathrm{~h}$ urine collections. Before analysis urine samples were hydrolysed using a 3 fold dilution with $0.2 \mathrm{~N} \mathrm{HCl}$, overnight incubation at room temperature and a further 10 fold diluting with a solution containing $6.5 \mathrm{~g} / \mathrm{dL}$ of bovine albumin in a phosphate buffer at $\mathrm{pH} 7.4$.

\subsubsection{External Quality Assessment (EQA) scheme}

The "Immunocheck" External Quality Assessment (EQA) scheme, conducted by QualiMedLab-CNR (Pisa, Italy) in co-operation with ProBioQual (Lyon, France), was established since the beginning of the 80s. QualiMedLab is a spin-off of the Italian Research Council (CNR) and operate together with the Institute of Clinical Physiology of the CNR and the Fondazione G. Monasterio in Pisa, Italy. Details of how QualiMedLab operates can be found on its website (http://www. qualimedlab.it). In particular, the 90 European participant laboratories to 2013 EQA cycle produced 726 aldosterone measurements (including 55 Italian laboratories), while the 111 participant laboratories to 2014 EQA cycle produced 980 aldosterone measurements (including 67 Italian laboratories).

For the preparation of study samples, several EDTA plasma samples were pooled together to obtain a sample pool with final volume of about $100 \mathrm{~mL}$, which was immediately stored at $-20^{\circ} \mathrm{C}$. All samples were tested for the absence of $\mathrm{HBsAg}$, antiHCV, and antiHIV. Plasma pools were prepared using residuals from samples collected from apparently healthy subjects and patients with cardiovascular diseases, collected in the Laboratory of the Fondazione G. Monasterio. Blood samples collected from approximately 30-50 subjects/patients were included in every study sample. A different pool was created for each control sample using plasma with similar aldosterone concentration. Subjects and patients gave the informed consensus for the use of their residual blood samples in the study.

Study samples were sent by mail as lyophilized materials. Lyophilization procedure was performed by Polymed (Sambuca, Firenze, Italy) within two weeks after preparation of sample pools. Stored sample pools, were defrozen, then distributed in approximately 200 vials (each containing a plasma/serum volume of $3.0 \mathrm{~mL}$ ), and finally lyophilized. Plasma aldosterone concentrations of all the study samples were measured before and after the lyophilization by the reference laboratory in order to evaluate the recovery of lyophilization procedure and the stability of aldosterone in the matrix samples. Samples with unreliable results, exceeding 3 standard deviations, were discarded.

\subsection{Statistical analysis}

Standard statistical analyses were performed using the Stat-View 5.0.1 program (1992-98, SAS Institute Inc., SSA Campus Drive, Cary, NC, USA). Both parametric and not parametric analyses were performed, the Tukey test was used for mean comparison.

The statistical analysis for EQA study was performed by QualiMedLab (http://www.ifc.cnr.it/eqas/). Total variability of the results obtained in the EQA study was estimated by averaging the CVs computed from the results of each study sample. This variability includes both systematic between-methods differences and differences introduced by the laboratories. The imprecision of the methods was estimated by averaging the CVs of the results produced by participants (using the same method) for the same study sample. Therefore, the reported average CVs, used for the calculation of imprecision profiles, were an estimate of the within-method, between-laboratories imprecision achieved by the method during the multicenter collaborative study. The consensus mean was calculated as the mean value of the all aldosterone concentrations measured by all participant laboratories in the same control samples. The values are reported as a mean $\pm S D$, if not otherwise stated.

\subsection{Results}

\subsubsection{Analytical performance of automated immunoassay methods}

In Table 1 data obtained for analytical sensitivity are showed. For the LIAISON method, the LoB value was calculated by considering the mean RLU value (132523 RLU, SD = 9339 RLU) obtained by repeatedly measuring $(n=65)$ a sample containing saline solution in 5 different runs using two different lots of calibrators. The RLU value of the 1.645 SD (i.e., $117161 \mathrm{RLU})$ was then interpolated on the mean standard curve of LIAISON method ( $R L U=131,791.174-812.374$ aldosterone, $\mathrm{R}=$ $0.947, \mathrm{n}=125$ ) to obtain the respective LoB value: $50.0 \mathrm{pmol} / \mathrm{L}$ of aldosterone (Table 1). The LoD was calculated according to the formula: $\mathrm{LoD}=\mathrm{LoB}+1.645 \mathrm{SD}$, using a plasma sample with very low aldosterone concentration (mean concentration $65.6 \mathrm{pmol} / \mathrm{L}, \mathrm{CV}=31.2 \%$, $\mathrm{n}=20$ ). As a result, the calculated LoD value was $83.9 \mathrm{pmol} / \mathrm{L}$, respectively (Table 1). For iSYS aldosterone concentrations of $53.1 \mathrm{pmol} / \mathrm{L}$ for LoB, $92.2 \mathrm{pmol} / \mathrm{L}$ for LoD, and $104.4 \mathrm{pmol} / \mathrm{L}$ for LoQ value at $20 \% \mathrm{CV}$ were respectively found (Table 1) [16]. The assay reproducibility of the aldosterone assay, evaluated in accordance with the CLSI EP5-A2 protocol [17] by repeatedly measuring 2 different EDTA plasma samples. Using LIAISON platform and samples containing on average $180.8 \mathrm{pmol} / \mathrm{L}$ (sample A) and $387.2 \mathrm{pmol} / \mathrm{L}$ (sample B) of aldosterone respectively were $12.9 \mathrm{CV} \%$ and $14.1 \mathrm{CV} \%$ for the sample A and 8.1 $\mathrm{CV} \%$ and $10.1 \mathrm{CV} \%$ for sample $\mathrm{B}$, for within-run and total imprecisions respectively. Using iSYS and samples containing on average $401.7 \mathrm{pmol} / \mathrm{L}$ (sample C) and $1293.7 \mathrm{pmol} / \mathrm{L}$ (sample D) of aldosterone respectively were $8.4 \mathrm{CV} \%$ and $12.7 \mathrm{CV}$ \% for the sample $\mathrm{C}$ and $5.5 \mathrm{CV} \%$ and $6.2 \mathrm{CV} \%$ for sample $\mathrm{D}$, for within-run and total imprecisions respectively.

The between-runs imprecision profiles of the two aldosterone immunoassay methods are reported in Fig. 1. The calculated limits of quantitation (LoQ) [15] at $20 \% \mathrm{CV}$ and $10 \% \mathrm{CV}$ were $111 \mathrm{pmol} / \mathrm{L}$ and $264 \mathrm{pmol} / \mathrm{L}$ of aldosterone, respectively using LIAISON platform, while the LoQ limits at $20 \% \mathrm{CV}$ and $10 \% \mathrm{CV}$ for the iSYS method were $104 \mathrm{pmol} / \mathrm{L}$ and $208 \mathrm{pmol} / \mathrm{L}$.

Both automated methods for aldosterone showed a close linearity to dilution test. These tests were performed in the two referral laboratories

\section{Table 1}

Comparison of analytical sensitivity parameters of the immunoassay methods for plasma aldosterone using the LIAISON and iSYS platforms.

\begin{tabular}{llll}
\hline Methods & LoB, pmol/L & LoD, pmol/L & LoQ at 20\% CV, pmol/L \\
\hline LIAISON platform & 50.0 & 83.9 & 111.1 \\
iSYS platform & 53.1 & 92.2 & 104.4 \\
\hline
\end{tabular}




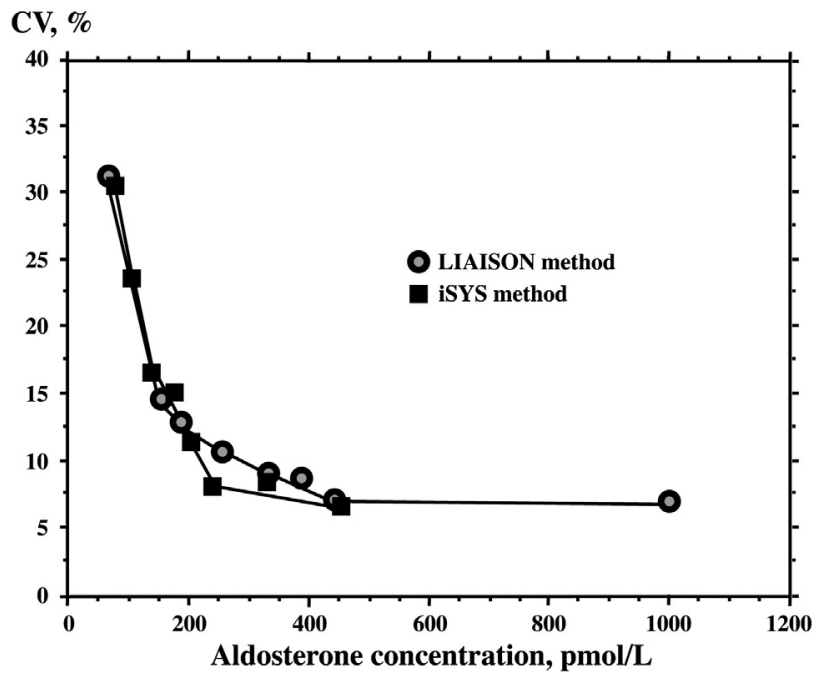

Fig. 1. Comparison of imprecision profiles of the two automated methods for aldosterone. The imprecision profiles of the two automated LIAISON and iSYS methods are shown in the figure. The calculated equations by means of the power regression analysis were $\mathrm{y}=152.7 x^{-0.571}$ and $\mathrm{y}=557.4 x^{-0.888}$ for the LIAISON and iSYS methods, respectively.

at least with two different plasma samples containing different aldosterone concentrations. The linearity of the immunoassays methods was evaluated according to the CLSI EP6-A protocol [18].

\subsubsection{Comparison of results obtained with different immunoassay methods}

In the referral Laboratory of Fondazione G. Monasterio, the aldosterone values measured with the LIAISON platform were compared to those found with iSYS platform in 290 plasma samples of 91 healthy subjects and 199 patients with cardiovascular diseases. These two immunoassay systems showed a close linear regression (LIAISON system $=71.9(95 \% \mathrm{CI}: 52.8-91.0)+0.873(95 \% \mathrm{CI}: 0.826-0.920)$ iSYS system; $R=0.906$ ) (Fig. 2A). A significant bias was found between the aldosterone values measured with these two methods (mean difference LIAISON - iSYS $=37.8 \pm 129.1 \mathrm{pmol} / \mathrm{L}$; Wilcoxon Signed Rank Test $\mathrm{p}<0.0001)$. The relationship between the percent difference of the two methods [(LIAISON - iSYS) / mean concentration $\times 100$ ] and the mean aldosterone concentration were reported in Fig. 2B. These data indicate that there is a significant bias $(p=0.0146)$ between these two methods, which proportionally increases with the aldosterone concentration, showing on average a difference of $15.0 \pm 71.8 \%$. However, the linear regression between the difference between the two methods and the mean aldosterone concentration measured by the two methods is not statistically significant (mean difference $=$ $37.8 \pm 129.1 \mathrm{pmol} / \mathrm{L} ; \mathrm{R}=0.087, \mathrm{p}>0.10$ ).

The correlations between the aldosterone concentrations measured with the two automated methods and some RIA methods by the referral laboratories were also evaluated. The results obtained with the LIAISON method in 314 plasma EDTA samples of healthy subjects and patients with cardiovascular disease were compared to those found with the RIA method, used for clinical routine in the laboratory. The results obtained by these two methods, ranging from 181.7 to $2177.8 \mathrm{pmol} / \mathrm{L}$, were significantly correlated (LIAISON method $=-15.8 \mathrm{pmol} / \mathrm{L}$ (95\%CI: from -48.5 to $16.9 \mathrm{pmol} / \mathrm{L})+0.779$ (95\%CI: $0.724-0.834$ ) RIA method, $\mathrm{R}=0.876$ ); however, LIAISON method showed on average lower aldosterone values compared to RIA method of about - 11.2\% (SD $118.2 \%, p<0.0001$ by Wilcoxon Signed Rank test). Moreover, a close linear regression was found between IDS-iSYS and Siemens Coat-A-Count RIA results in 220 EDTA plasma samples [iSYS method $=-0.92(95 \% \mathrm{CI}$ : $-1.98-0.23)+1.26$ (95\%CI: $1.17-1.36)$ RIA Siemens, $R=0.95]$. In the Bland-Altman plot the mean was $-9.4 \mathrm{pmol} / \mathrm{L}$ with the range between $\pm 1.96 \mathrm{SD}$ from -44.4 to $25.6 \mathrm{pmol} / \mathrm{L}$. The aldosterone concentrations measured with iSYS platform were also found to be closely

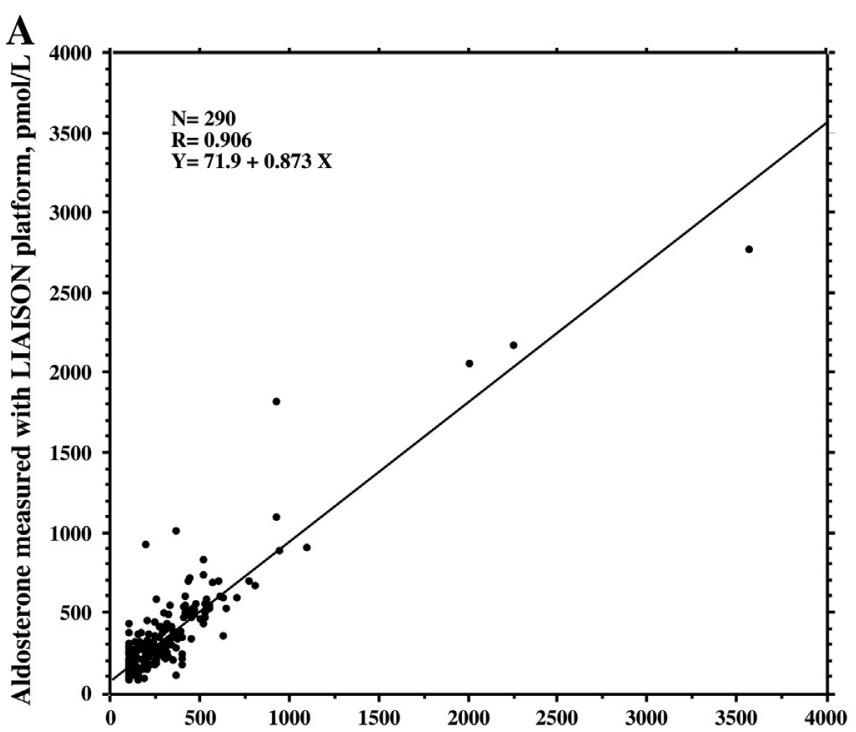

Aldosterone measured with iSYS platform, pmol/L

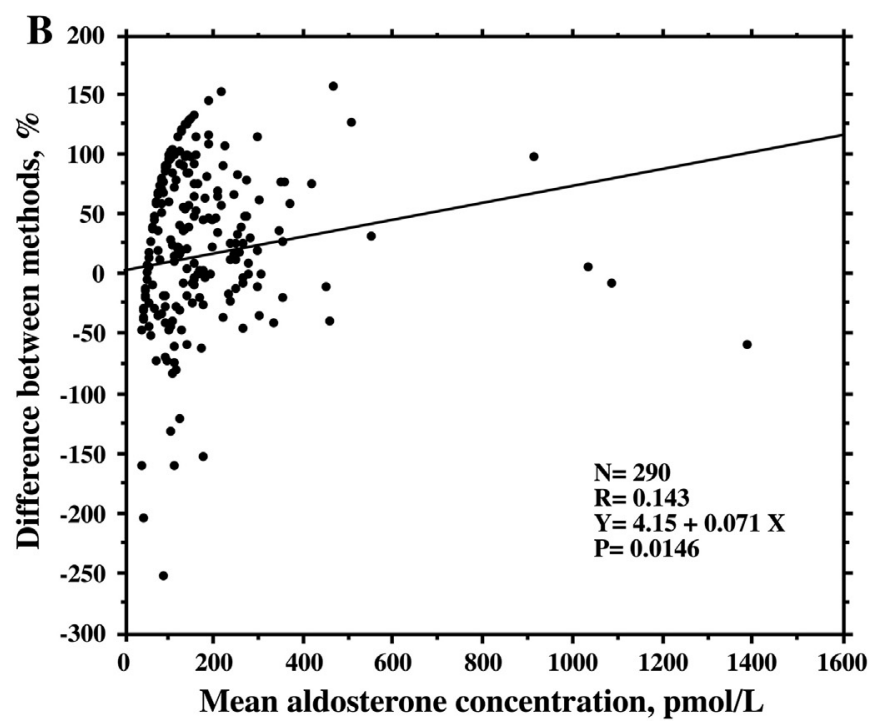

Fig. 2. Comparison of the aldosterone concentrations measured with LIAISON and iSYS platforms. Part A. Linear regression between the aldosterone concentrations measured by iSYS platform (X axis) and LIAISON platform (Y axis) in 290 plasma samples of 91 healthy subjects and 199 patients with cardiovascular diseases. Part B. Linear relationship between the percent difference between the aldosterone concentrations measured by the two automated methods [(LIAISON - iSYS) / mean concentration $\times 100$ ], reported in the Y-axis, and the mean aldosterone concentrations measured in the same plasma sample using the two automated platforms, reported in the $\mathrm{X}$-axis.

correlated to those obtained with the ALDOCTK-2 RIA [iSYS method $=-53.7$ (95\% CI: from -84.1 to -33.9$)+0.78(95 \% \mathrm{CI}$ : 0.71-0.84) RIA DiaSorin, $\mathrm{R}=0.93$ ]. In Bland-Altman plot the mean was $40.7 \mathrm{pmol} / \mathrm{L}$ with the range between $\pm 1.96 \mathrm{SD}$ from -38.0 to $119.4 \mathrm{pmol} / \mathrm{L}$.

Finally, in the laboratory of "San Bortolo" Hospital, aldosterone concentrations ranged from 50.0 to $1125 \mathrm{pmol} / \mathrm{L}$ in 88 urine samples; the Passing-Bablok regression of iSYS against RIA DiaSorin shows a slope of 1.20 (95\%Cl: 1.11-1.28) and intercept 2.1 (95\%Cl:1.08-3.19). The obtained Bland-Altman \%mean ( +1.96 SD range) was -41 ( -99.8 to 20.3). In the comparison between methods all results obtained from the instruments were considered for regression parameters, also if the aldosterone concentrations were below of the LoD values calculated in the present study. 


\subsubsection{Results of the External Quality Assessment (EQA) study}

In Table 2, the aldosterone values measured with the two automated systems by the two reference laboratories in 12 control samples distributed in the annual 2013 cycle of the EQA Immunocheck study were reported. For comparison, the aldosterone values measured in the same control samples by the participant laboratories to the EQA study using some RIA and EIA methods were also reported in Table 2. Furthermore, the consensus means, which is the mean calculated considering all the aldosterone concentrations measured by the participant laboratories in the same sample after the exclusion of outlier values, were also reported in Table 2. In Table 3, the correlation matrix between the aldosterone values measured with RIA and EIA methods by laboratories participant to the EQA and with the two automated platform by the reference laboratories were reported. All methods, with the exclusion of the RIA method by DRG Instruments GMGH, were closely correlated between themselves and with consensus mean values. In particular, the results obtained with the two automated immunoassay methods tested in the present study were highly correlated with consensus mean values (for LIAISON method, $R=0.964, p<0.001$, and for iDYS method, $\mathrm{R}=0.990, \mathrm{p}<0.001)$. Moreover, the mean aldosterone values measured with LIAISON method (367.2 $\pm 198.9 \mathrm{pmol} / \mathrm{L})$ and iDYS method $(407.2 \pm 287.2 \mathrm{pmol} / \mathrm{L})$ were not significantly different $(\mathrm{p}=$ 0.2163 by paired $t$ test).

\subsection{Clinical results}

Aldosterone was measured with the LIAISON platform in 168 plasma samples of adult healthy subjects (65 men, age $48.0 \pm 11.6$ years; 103 women, age $48.0 \pm 11.3$ years). Considering the whole population, the aldosterone concentrations corresponding to mean, median, interquartile range, and 97.5th percentile values were $271.6 \mathrm{pmol} / \mathrm{L}$, $234.9 \mathrm{pmol} / \mathrm{L}, 173.1-326.9 \mathrm{pmol} / \mathrm{L}$, and $639.6 \mathrm{pmol} / \mathrm{L}$, respectively. Moreover, a significant difference ( $\mathrm{p}=0.0144$ by Mann-Whitney $\mathrm{U}$ test) was found between the mean values of plasma aldosterone found in women $(290.6 \pm 160.1 \mathrm{pmol} / \mathrm{L}$, median $254.6 \mathrm{pmol} / \mathrm{L})$ and in men $(241.3 \pm 134.6 \mathrm{pmol} / \mathrm{L}$, median $203.9 \mathrm{pmol} / \mathrm{L})$, respectively. Finally, a significant negative correlation was found between age and plasma aldosterone values ( $\mathrm{Rho}=-0.238, \mathrm{p}=0.002$ by Spearman Rank Correlation test).

Blood samples were also drawn from 20 healthy subjects in a standing or supine position, aged 21-65 years. The standing samples were drawn after the subjects had been walking for $2 \mathrm{~h}$, and the supine

Table 2

Aldosterone values measured by participant laboratories to EQA study in the 12 study samples.

\begin{tabular}{lllllrrrr}
\hline Study sample & CM & SOR & IMT & \multicolumn{1}{c}{ LSN } & \multicolumn{1}{c}{ DRG } & \multicolumn{1}{c}{ DPC } & \multicolumn{1}{c}{ DSL } & IDSY \\
\hline Sample 01, pmol/L & 107.5 & 181.9 & 48.6 & 171.1 & 90.8 & 86.9 & 93.1 & 157.2 \\
Sample 02, pmol/L & 119.4 & 186.7 & 71.9 & 149.2 & 109.7 & 81.1 & 141.1 & 123.6 \\
Sample 03, pmol/L & 200.3 & 256.7 & 176.1 & 189.4 & 114.4 & 121.1 & 189.7 & 123.1 \\
Sample 04, pmol/L & 211.1 & 269.4 & - & 210.3 & 118.9 & 126.4 & 217.2 & 166.9 \\
Sample 05, pmol/L & 238.3 & 371.4 & 156.9 & 300.6 & 180.6 & 245.8 & 319.2 & 230.8 \\
Sample 06, pmol/L & 262.5 & 374.4 & 163.6 & 292.5 & 323.6 & 245.6 & 273.6 & 259.2 \\
Sample 07, pmol/L & 330.8 & 454.4 & 331.7 & 296.1 & 199.7 & 305.3 & 298.9 & 335.0 \\
Sample 08, pmol/L & 340.8 & 454.7 & 372.2 & 309.7 & 236.4 & 415.8 & 325.0 & 372.8 \\
Sample 09, pmol/L & 630.8 & 844.7 & 541.4 & 660.3 & 243.3 & 634.4 & 725.0 & 722.5 \\
Sample 10, pmol/L & 662.8 & 861.1 & 566.7 & 704.2 & 265.3 & 607.8 & 764.2 & 828.1 \\
Sample 11, pmol/L & 674.2 & 857.2 & 657.2 & 563.1 & 420.3 & 549.4 & 645.6 & 780.6 \\
Sample 12, pmol/L & 690.6 & 807.5 & 832.8 & 559.2 & 680.0 & 516.4 & 637.5 & 795.0 \\
Number of results & 701 & 180 & 169 & 80 & 62 & 52 & 51 & 36
\end{tabular}

CM: consensus mean; SOR: ALDOCTK-2 RIA (DiaSorin, Saluggia, Italy); IMT: Immunotech/ Beckman Coulter RIA (distributed in Italy by Pantec Srl, Torino); LSN: LIAISON automated platform (DiaSorin, Saluggia, Italy); DRG: Aldosterone ELISA (DRG Instruments GMGH, Marburg, Germany, distributed in Italy by Pantec Srl, Torino); DPC: Coat-A-Count Aldosterone RIA (DPC Biermann GmbH, Bad Nauheim, Germany, distributed by Siemens Health Care Diagnostics, Germany); DSL: Aldosterone RIA (Diagnostic System Laboratories, Inc; Webster, USA, distribute in Italy by Pantec Srl, Torino); IDSY: iSYS automated platform (IDS Ltd., Boldon, UK).
Table 3

Correlation matrix between consensus mean and plasma aldosterone values measured with the immunoassay methods used by participant laboratories in the 12 control study samples distributed in the EQA study.

\begin{tabular}{lllllllll}
\hline & CM & SOR & IMT & LSN & DRG & DPC & DSL & IDSY \\
\hline CM & 1.000 & 0.994 & 0.966 & 0.964 & 0.759 & 0.958 & 0.977 & 0.990 \\
SOR & 0.994 & 1.000 & 0.937 & 0.981 & 0.710 & 0.976 & 0.988 & 0.989 \\
IMT & 0.966 & 0.937 & 1.000 & 0.875 & 0.845 & 0.896 & 0.899 & 0.947 \\
LSN & 0.964 & 0.981 & 0.875 & 1.000 & 0.626 & 0.965 & 0.994 & 0.972 \\
DRG & 0.759 & 0.710 & 0.845 & 0.626 & 1.000 & 0.645 & 0.652 & 0.738 \\
DPC & 0.958 & 0.976 & 0.896 & 0.965 & 0.645 & 1.000 & 0.966 & 0.958 \\
DSL & 0.977 & 0.988 & 0.899 & 0.994 & 0.652 & 0.966 & 1.000 & 0.974 \\
IDSY & 0.990 & 0.989 & 0.947 & 0.972 & 0.738 & 0.958 & 0.974 & 1.000 \\
\hline
\end{tabular}

Abbreviations are identical to those of Table 2.

samples were drawn after the subjects had been lying supine for at least $1 \mathrm{~h}$. The key parameters describing the distributions of results obtained with iSYS and LIAISON methods are reported in Fig. 3 and Table 4. Interestingly, there was a significant difference between the aldosterone values obtained in supine position with the two methods ( $\mathrm{p}<0.0001$ by paired $t$ test), while the aldosterone values observed in standing position were not significantly different $(\mathrm{p}=0.327)$.

\subsection{Discussion}

RIA methods, commonly used for the measurement of aldosterone in blood or urine samples, actually show some critical analytical issues, such as long incubation times (up to $18 \mathrm{~h}$ ), the need to assay several samples together in the same run in order to minimize the cost, the relative short expiration date of radiolabeled reagents (usually less than 60 days), and the drawbacks related to the use of radioactive materials. Our data demonstrate that the new competitive immunoassays for aldosterone using LIAISON and iSYS platforms actually show better analytical characteristics compared to RIA methods. In particular, these fully automated methods can assay few samples in less than an hour (TAT about $40 \mathrm{~min}$ ), and the expiration date for reagents and materials is usually more than 9 months for both methods. Furthermore, the results reported in Tables 2 and 3 demonstrated that the immunoassays using LIAISON and iSYS platforms showed aldosterone values highly correlated with the most popular RIA and EIA methods used in the EQA study, as well as the consensus mean values obtained in this multicenter study.

Moreover, the results of the present study indicate that the new competitive immunoassays for aldosterone using LIAISON and iSYS platforms showed similar analytical characteristics, such as analytical sensitivity parameters (Table 1) and imprecision profiles (Fig. 1). Moreover, the aldosterone values measured in 12 control samples with these two methods were closely correlated with a mean difference of $10.3 \%$ (mean difference, $40.0 \mathrm{pmol} / \mathrm{L} /$ mean concentration, $387.2 \mathrm{pmol} / \mathrm{L}, \times 100$ ), which was not significantly different. On the other hand, a significant bias, which proportionally increases with the aldosterone concentration, was found between these two methods, when the aldosterone concentrations were measured in 290 plasma samples of 91 healthy subjects and 199 patients with cardiovascular diseases (Fig. 2B). The discrepancy between the results obtained with control EQA samples and plasma samples of healthy subjects and patients, may be due to different matrix effects (lyophilized materials vs plasma samples). However, the larger number of plasma samples assayed by referral laboratories (i.e., 290), compared to lower number of control samples measured by participant laboratories to EQA scheme (only 12), should take into account to explain these apparently conflicting results. Indeed, although we found a proportional bias between the two automated methods, which was statistically significant ( $\mathrm{p}=0.0146)$, on average the percent difference between methods was relatively low $(15.0 \pm 71.8 \%)$, and the linear correlation between the percent difference and the mean aldosterone concentration values, measured with the two methods, was also relatively poor $(R=0.143)$ (Fig. 2B). These 


\begin{tabular}{lcccccccc}
\hline & Mean & $\mathbf{9 5 \%} \mathbf{C l}$ & Median & $\mathbf{9 5 \%} \mathbf{C l}$ & $\mathbf{2 . 5}-\mathbf{9 7 . 5}$ P.le & $\mathbf{5}$ - $\mathbf{9 5}$ P.le & $\mathbf{1 0 - 9 0}$ P.le & $\mathbf{2 5}-\mathbf{7 5}$ P.le \\
isYS & 321,1 & $280,0-362,3$ & 291,7 & $245,6-315,5$ & $127,8-842,9$ & $137,1-611,9$ & $139,4-530,6$ & $195,8-401,4$ \\
Liaison & 324,9 & $289,5-360,3$ & 281,9 & $248,4-313,5$ & $89,6-773,3$ & $113,4-612,8$ & $155,9-556,9$ & $203,2-413,9$ \\
\hline
\end{tabular}

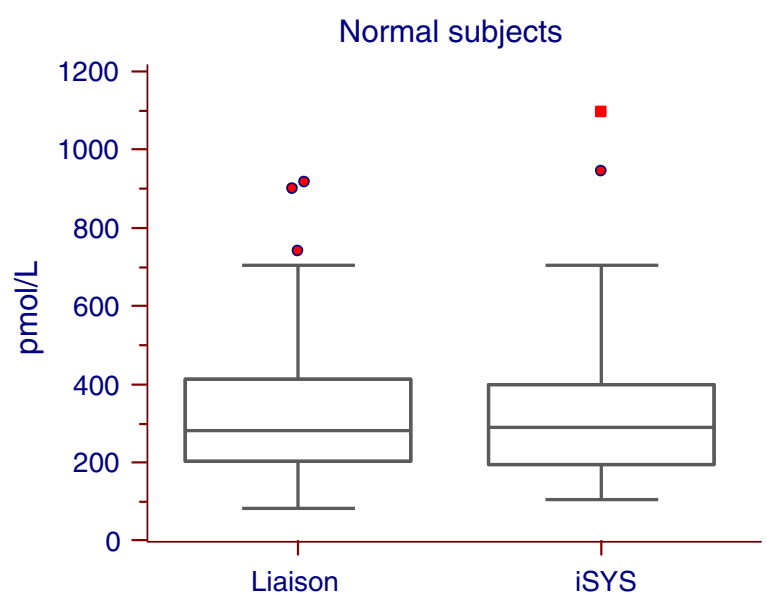

Fig. 3. Box plot and key parameters representing the comparison of aldosterone concentrations obtained with LIAISON and iSYS methods.

data support the hypothesis that these conflicting results are probably due to the large number of plasma samples tested rather than the matrix effects.

A relative limitation of this study is that different experiments were performed (i.e., different replicates and number of samples) in the two different referral laboratories, in particular when assessing performance parameters, such as LoD, LoQ and imprecision. On the other hand, the strength of this study is the large number of experimental plasma samples tested (up to 290 in some evaluations) (Fig. 2) and the use of EQA scheme results, including some hundreds of aldosterone determinations performed by about 100 clinical laboratories using the most popular commercial aldosterone immunoassays in Europe (Tables 2 and 3). This huge lot of data actually guaranteed robust statistical evaluations.

At present time, the measurement of aldosterone in plasma or urine samples is recommended by international guidelines only for screening of primary aldosteronism in patients at high risk for this clinical condition $[3,4]$. However, excessive tissue production of aldosterone occurs in cardiovascular diseases, including myocardial infarction and heart failure, resulting in a multitude of adverse effects in the cardiovascular system necessitating pharmacologic blockade of this steroid hormone [19]. Both human and animal studies have consistently proven the beneficial effects of aldosterone antagonist treatment on: 1) endothelial function, 2) modulation of inflammatory mechanisms between blood and the vascular wall and 3 ) reduction of tissue proliferation and cardiovascular remodeling leading to different severities of cardiovascular damage [19]. According to these basic mechanisms of anti-aldosterone therapy, some clinical trials reported some beneficial effects of aldosterone blockers in cardiovascular diseases, in particular in heart failure patients, as recently reviewed [19-21]. More recently, several studies evaluated the usefulness of this type of intervention in preventing

Table 4

Distribution of supine and standing EDTA plasma aldosterone concentrations ( $\mathrm{pmol} / \mathrm{L}$ ) in 20 healthy volunteers.

\begin{tabular}{lllrrrrl}
\hline \multirow{2}{*}{ Method } & Position & N & Mean & \multicolumn{1}{l}{$95 \% \mathrm{Cl}$} & Median & Minimum & Maximum \\
\hline \multirow{2}{*}{ LiaisonXL } & Supine & 20 & 175.8 & $154.7-196.7$ & 180.6 & 111.1 & 244.4 \\
& Upright & 20 & 333.6 & $276.9-390.3$ & 306.9 & 183.3 & 583.3 \\
\multirow{2}{*}{ iSYS } & Supine & 20 & 87.5 & $59.4-115.6$ & 72.2 & 25.0 & 183.3 \\
& Upright & 20 & 315.83 & $231.1-400.3$ & 291.7 & 105.6 & 797.2 \\
\hline
\end{tabular}

vascular and cardiac fibrosis, myocardial hypertrophy and remodeling in refractory hypertensive syndromes [22-24]. In these studies [22-24] aldosterone levels were not usually measured, probably because RIA methods are considered to be poorly suitable for large clinical trials. However, it is conceivable that monitoring of aldosterone concentrations may be useful in patients with heart failure. Indeed, it is well known that cornerstone drugs for management of heart failure, such as ACE-inhibitors or angiotensin receptor blockers, may affect aldosterone production or, as the case of aldosterone receptor blockers, antagonize its biological effects. In particular, after an initial decline, a stable increase in aldosterone plasma levels has been reported to occur in up to $40 \%$ of symptomatic heart failure patients on ACE-inhibitors or angiotensin receptor blockers [25]. This phenomenon was formerly termed 'aldosterone escape' by Pitt in 1995 [26], and recently renamed as 'aldosterone breakthrough', to avoid the confusion with the "escape" from the sodium-retaining effects of excess mineralocorticoids or aldosterone in primary hyperaldosteronism. Aldosterone breakthrough is clinically relevant, given aldosterone promotion of oxidative stress, endothelial dysfunction, inflammation and fibrosis, leading to cardiovascular and renal injury. Indeed, a reliable measure of the aldosterone could hold clinical relevance also in the heart failure setting.

\subsection{Conclusions}

In conclusion, our data indicate that the two new automated methods, evaluated in the present study, may be more suitable for monitoring aldosterone levels in patients with cardiovascular diseases than RIA methods, due to their better analytical performance in terms of reproducibility and a shorter TAT. However, in our study significant bias was observed in result comparison, this means that translating aldosterone concentration in clinical information an appropriate definition of reference ranges for each method is mandatory.

\section{Authorship}

All authors listed have participated in either the conception or planning of the work, the interpretation of the results and the writing of the paper. 


\section{Disclosure summary}

None of authors has potential conflict of interest to be declared. Clinical Trial Registration Number: not applicable.

\section{Acknowledgments}

We thank DiaSorin and IDS for providing reagents needed for this study without charge and without interfering in data evaluation.

\section{References}

[1] Williams GH. Aldosterone biosynthesis, regulation, and classical mechanism of action. Heart Fail Rev 2005;10:7-13.

[2] Knox F, Burnett JJ, Kohan D, Kohan DE, Spielman WS, Strand JC. Escape from the sodium-retaining effects of mineralocorticoids. Kidney Int 1980;17:263-76.

[3] Funder JW, Carey RM, Fardella C, et al. Case detection, diagnosis, and treatment of patients with primary aldosteronism: an Endocrine Society Clinical Practice Guideline. J Clin Endocrinol Metab 2008;93:3266-81.

[4] Giacchetti G, Mulatero P, Mantero F, Veglio F, Boscaro M, Fallo F. Primary aldosteronism, a major form of low renin hypertension: from screening to diagnosis. Trends Endocrinol Metab 2008;19:104-8.

[5] Bayard F, Beitins I, Kowarski A, Migeaon C. Measurement of plasma aldosterone by radioimmunoassay. J Clin Endocrinol Metab 1970;31:1-6.

[6] Miller M, Sagnella G, MacGregor G. Extraction method and nonex-tracted kit method compared for measuring plasma aldosterone. Clin Chem 1997;43:1995-7.

[7] Schirpenbach C, Seiler L, Maser-Gluth C, Beuschlein F, Reincke M, Bidlingmaier M. Automated chemiluminescence-immunoassay for aldosterone during dynamic testing: comparison to radioimmunoassays with and without extraction steps. Clin Chem 2006;52:1749-55.

[8] Manolopoulou J, Mulatero P, Maser-Gluthc C, et al. Saliva as a medium for aldosterone measurement in repeated sampling studies. Steroids 2009;74:853-8.

[9] Walsh PR, Wang MC, Turner EA. Non-chromatographic radioimmunoassay procedure for urinary aldosterone. Clin Chem 1979;25:1226-9.

[10] Al-Dujaili EA, Edwards CR. Development and application of a simple radioimmunoassay for urinary aldosterone. Clin Chim Acta 1981;116:277-87.

[11] Stöckl D, Reinauer H, Thienpont LM, De Leenheer AP. Determination of aldosterone in human serum by isotope dilution gas chromatography/mass spectrometry using a new heptafluorobutyryl derivative. Biol Mass Spectrom 1991;20:657-64.

[12] Thienpont LM, Van Nieuwenhove B, Stöckl D, Reinauer H, De Leenheer AP. Determination of reference method values by isotope dilution-gas chromatography/mass spectrometry: a five years' experience of two European Reference Laboratories. Eur J Clin Chem Clin Biochem 1996;34:853-60.

[13] Taylor PJ, Cooper PD, Gordon RD, Michael Stowasser M. Measurement of aldosterone in human plasma by semiautomated HPLC-tandem mass spectrometry. Clin Chem 2009;55:1155-62.

[14] LIAISON Aldosterone (310450). Instructions for use. Performance evaluation only, 310450, 12/2012. Stillwater, USA: DiaSorin Inc.; 2012 1-7.

[15] IDS-iSYS Aldosterone REF IS-3300. Instructions for use. IS-3300PL, V02, 2012-06-08 Tyne \& Wear, England: Immunodiagnostic System Ltd (IDS Ltd); 2012 1-4.

[16] CLSI EP17-A protocol. Protocols for determination of limits of detection and limits of quantitation; approved guidelines, vol. 24 (No. 34). Wayne, Pennsylvania: CLSI NCCLS; 2004

[17] CLSI EP5-A2 protocol. Evaluation of precision performance of quantitative measurement methods; Approved guideline, vol. 24 (No. 25). Wayne, Pennsylvania: CLS NCCLS; 2004.

[18] CLSI EP6-A protocol. Evaluation of the linearity of quantitative measurement procedures: A statistical approach; Approved guideline, vol. 23 (No 16). Wayne, Pennsylvania: CLSI NCCLS; 2003.

[19] Martinez FA. Aldosterone inhibition and cardiovascular protection: more important than it once appeared. Cardiovasc Drugs Ther 2010;24:345-50.

[20] Rous GC, Kaur R, Ernst M. Diuretics: a review and update. J Cardiovasc Pharmaco Ther 2014:19:5-13.

[21] van den Berg TN, Rongen GA, Fröhlich GM, Deinum J, Hausenloy DJ, Riksen NP. The cardioprotective effects of mineralocorticoid receptor antagonists. Pharmacol Ther Nov 23 2013. http://dx.doi.org/10.1016/j.pharmthera. 2013.11.006 [pii: S01637258(13)00224-6, Epub ahead of print].

[22] Boccanelli A, Mureddu GF, Cacciatore G, et al. Anti-remodelling effect of canrenone in patients with mild chronic heart failure (AREA IN-CHF study): final results. Eur J Heart Fail 2009;11:68-76.

[23] Kasama S, Toyama T, Sumino H, et al. Effects of mineralocorticoid receptor antagonist spironolactone on cardiac sympathetic nerve activity and prognosis in patients with chronic heart failure. Int J Cardiol 2013;167:244-9.

[24] Shah AM, Shah SJ, Anand IS, et al. Cardiac structure and function in heart failure with preserved ejection fraction: baseline findings from the echocardiographic study of the treatment of preserved cardiac function heart failure with an aldosterone antagonist trial. Circ Heart Fail 2014;7:104-15.

[25] Lee AF, MacFadyen RJ, Struthers AD. Neurohormonal reactivation in heart failure patients on chronic ACE inhibitor therapy: a longitudinal study. Eur J Heart Fail 1999;1: 401-6.

[26] Pitt B. "Escape" of aldosterone production in patients with left ventricular dysfunction treated with an angiotensin converting enzyme inhibitor: implications for therapy. Cardiovasc Drugs Ther 1995;9:145-9. 